\title{
Patient-centric dose equivalency pilot study of incobotulinumtoxin a (xeomin) vs. abobotulinumtoxin a (dysport) in the treatment of glabellar frown lines
}

\author{
Jonathan Bank ${ }^{1}$, Nicole A. Phillips ${ }^{2}$, Laurie A. Casas ${ }^{1}$ \\ ${ }^{1}$ Department of Surgery, Section of Plastic and Reconstructive Surgery, University of Chicago Medical Center, Chicago, IL 60026, USA. \\ ${ }^{2}$ Department of Surgery, Division of Plastic Surgery, Brigham and Women's Hospital, Boston, MA 02115, USA.
}

Address for correspondence: Dr. Laurie A. Casas, Department of Surgery, Section of Plastic and Reconstructive Surgery, University of Chicago Medical Center, Chicago, IL 60026, USA. E-mail: lcasas@casas.md

\begin{abstract}
Aim: Incobotulinumtoxin A (xeomin) has been proposed as an alternative to abobotulinumtoxin A (dysport) and onabotulinumtoxin A (Botox) in the treatment of glabellar frown lines. A recent study is comparing abobotulinumtoxin A and onabotulinumtoxin A revealed equivalent efficacy with a dose conversion ratio of 2.5:1. We sought to establish effectiveness and dosing equivalency of incobotulinumtoxin A vs. abobotulinumtoxin A. Methods: Inclusion criteria for this pilot study included patients of a single surgeon (LAC) who had previously received a constant dose of abobotulinumtoxin A over at least four consecutive treatment sessions for the previous 12 months to achieve an $85-90 \%$ elimination of dynamic glabellar frown lines. The primary outcome sought dose comparison between established maintenance abobotulinumtoxin A dosing and incobotulinumtoxin A first-time dosing. A 2:1 conversion (abobotulinumtoxin A: incobotulinumtoxin A) was chosen in most patients. Secondary outcomes were patient-reported onset of effect, physician-assessed effect at 10-12 weeks, pain associated with administration, and patient perceived need for re-treatment at 2 weeks. Results: A total of 32 subjects were included. The mean dose of incobotulinumtoxin A was 17.1 units $( \pm 6.1$, the median dose 20 units). The mean dose of abobotulinumtoxin A was $27.6( \pm 11.7$, the median dose 27.5 units). The mean difference in treatment units was -10.5 (95\% confidence interval, $P<0.001$ ). Among 30 patients who reported effect onset, the median was 8.5 days, with a range of 1-14. At 10-12 weeks, muscle paralysis was assessed to be $69.2 \%( \pm 27.3), v$ s. $90.3 \%( \pm 1.8)$ with abobotulinumtoxin A $(P<0.001)$. The majority of patients rated pain of administration as equal or greater to that of abobotulinumtoxin A (63\% and $22 \%$, respectively). Three patients $(9 \%)$ required re-treatment at 2 weeks with abobotulinumtoxin A due to lack of effective treatment with incobotulinumtoxin A. Abobotulinumtoxin A re-treatment was chosen by the patient. Conclusion: We found incobotulinumtoxin A at $17.1( \pm 6.1)$ units to be less effective than abobotulinumtoxin A at $27.6( \pm 11.7)$ units in the treatment of glabellar frown lines at 10-12 weeks postadministration. Dosing was less predictable than dosing associated with abobotulinumtoxin A treatment. Larger, randomized controlled trials are indicated to further delineate these differences and to clarify whether this difference from previously published incobotulinumtoxin A dosing may have been due to the small sample size.
\end{abstract}

\begin{tabular}{|l|l|}
\hline \multicolumn{2}{|c|}{ Access this article online } \\
\hline Quick Response Code: & Website: \\
\hline & www.parjournal.net \\
\cline { 2 - 2 } & \\
\hline
\end{tabular}

Key words:

Abobotulinumtoxin A, glabellar frown lines, incobotulinumtoxin A, rhytids 


\section{INTRODUCTION}

Neurotoxin injection accounted for more than a third of all nonsurgical cosmetic procedures performed in the United States in 2012.[1] Abobotulinumtoxin A (aboBTX-A, dysport) and onabotulinumtoxin A (onaBTX-A, Botox) are currently the most commonly used preparations of Clostridium botulinum toxin in the treatment of glabellar frown lines. Composed of high-molecular weight protein complexes unique to the various formulations, each of these neuromodulators has a slightly different pharmacokinetic profile. ${ }^{[2]}$ The protein complex is also thought to play a role in the immunogenicity of these therapeutic agents, influencing tolerance to drug effect and clinical response over time. ${ }^{[3]}$

Abobotulinumtoxin A (aboBTX-A, dysport) is a type A botulinum toxin approved for the temporary improvement in the appearance of moderate to severe glabellar lines associated with procerus and corrugator muscle activity. ${ }^{[4]}$ The toxin exists in a protein complex approximately $500 \mathrm{kDa}$ in size. Incobotulinumtoxin A (incoBTX-A, xeomin), a recently approved form of botulinum toxin type $A$, is also indicated for the temporary improvement in the appearance of moderate to severe glabellar lines associated with corrugator and/ or procerus muscle activity in adult patients. ${ }^{[5]}$ IncoBTX-A differs from the other neuromodulators, including onaBTX-A and aboBTX-A, in its formulation as a purified toxin-free of complexing proteins. This formulation, in theory, renders incoBTX-A less immunogenic than the other forms of botulinum toxin A currently available, permitting reproducible effects on repeat injections.

Although a calculated unit conversion between alternative neurotoxin preparations cannot be directly derived due to differing manufacturing processes, the separate bacterial strains involved, and the variations in size of the associated complexing proteins, ${ }^{[2,6,7]}$ a published clinical study as well as the senior author's clinical experience has shown that a reliable conversion rate between aboBTX-A (dysport) and onaBTX-A (Botox) is 2.5:1. ${ }^{[8]}$ In addition, several studies have concluded that a $1: 1$ equivalency exists between onaBTX-A (Botox) and incoBTX-A (xeomin). ${ }^{[9,10]}$

In our practice since 2010, the majority of patients treated with neuromodulators receive aboBTX-A. Given the potentially promising immunogenic profile of incoBTX-A, ${ }^{[11]}$ we were interested in determining the correct dosage of this neuromodulator among our patient population. Due to the uncertainty regarding conjectural conversion between the three neurotoxin preparations, we sought to establish effectiveness and dosing equivalency of incoBTX-A versus aboBTX-A within a consecutive series of 32 patients previously treated with aboBTX-A for temporary reduction in glabellar dynamic wrinkles.

\section{METHODS}

We conducted a prospective pilot study at a single surgeon center, with all injections performed by the senior author. The study included patients that had previously received at least four consecutive treatments of aboBTX-A at 4-month intervals at a stable dose, achieving $85-90 \%$ elimination of dynamic glabellar frown lines, and who had expressed satisfaction with at least four consecutive aboBTX-A treatments. Patient satisfaction with aboBTX-A treatment was defined by the patient's decision not to return for re-treatments before the planned redosing interval of 4-month. The study was approved by the review board of University of Chicago Medical Center.

Primary outcomes of this pilot study were patient perceived clinical effectiveness at 2 weeks (defined as an $85-90 \%$ decrease in muscle activity) and percentage of muscle activity at 3 months per surgeon assessment. The planned conversion rate was 2:1 (aboBTX-A: incoBTX-A) using the established aboBTX-A dosage for each patient to determine an initial incoBTX-A dose. However, we permitted dose adjustment per the physician's assessment at the time of incoBTX-A injection. The incoBTX-A dose was dispersed into the corrugator and procerus muscles for each patient; injection patterns were based on diagrams reviewed in the electronic medical record, documenting the injection points used during prior aboBTX-A treatments.

Secondary outcomes were examined via patient questionnaires that asked subjects to report on the onset of effect after incoBTX-A injection, the pain of injection versus their recollection of pain on aboBTX-A injections, the perceived duration of effect, and their overall satisfaction with the treatment session. Patients who were not satisfied with their initial treatment dosage were asked to return for re-treatment at 2 weeks. All patients were seen in follow-up at 10-12 weeks. At this time, glabellar and procerus activity were assessed by the treating surgeon, and the patient's perception of the treatment was recorded.

Demographic and clinical characteristics were summarized using frequency counts and percentages for categorical variables and median and range for continuous variables. Residual muscle activity at 4-month following at least four abobotulinumtoxin A treatment sessions was compared to muscle activity with one incobotulinumtoxin A treatment session at 10-12 weeks using a paired $t$-test.

\section{RESULTS}

A total of 32 subjects were included. The majority of patients were female (40-71 years old) and most had received aboBTX-A consistently for over 2 years [Table 1]. The mean dose of incoBTX-A administered was 17.1 units ( \pm 6.1 , median dose 20 units). The mean treatment dose of aboBTX-A administered was $27.6( \pm 11.7$, median dose 27.5 units). The mean difference in treatment units was -10.5 (95\% confidence interval, $P<0.001)$ [Table 2]. Among 30 patients who reported effect onset of the one incoBTX-A treatment, the median result was 8.5 days, with a range of 1-14 days [Table 1].

Twenty-nine out of 32 patients $(91 \%)$ reported satisfactory treatment effect at 2 weeks, 3 patients $(9 \%)$ requested 
re-treatment at 2 weeks with aboBTX-A due to perceived lack of effective treatment with incoBTX-A. During follow-up with the treating surgeon at 10-12 weeks, muscle paralysis was assessed to be $69.2 \%$ ( \pm 27.3 ), vs. $90.3 \%( \pm 1.8)$ in an equivalent time period with aboBTX-A $(P<0.001)$ [Table 2]. With regard to perceived pain on injection, $62 \%$ of patients reported equivalent pain between the two treatments, $22 \%$ of patients reported more pain with the incoBTX-A injection, 9\% had less pain with incoBTX-A, and $6 \%$ were unable to reliably recall. Overall satisfaction with incoBTX-A treatment was confirmed by 22 out of 32 patients among the study group (68\%) at 2 weeks; this increased to 25/32 (78\%) at 3 months. The most commonly cited reasons for dissatisfaction were related to the longevity and magnitude of incoBTX-A's effect.

At 10-12 weeks following incoBTX-A treatment, the percentage of muscle activity was recorded by the senior surgeon in light of the dose ratio of aboBTX-A: incoBTX-A administered. Of the subjects that received an incoBTX-A dose at a ratio of $1.5-2.5: 1$, only $40 \%$ had a clinical result that was considered equivalent to the original four aboBTX-A treatment dose results. A total of $16 \%$ of patients had effects that were less than expected for the hypothesized ratio. Some patients (38\%) received relatively more incoBTX-A (ratio of $0.5-1.5: 1$ ), due to clinical evaluation that the dose should be higher than $2: 1$ of the original aboBTX-A dose. Among this study group, $16 \%$ resulted in muscle attenuation that was greater than

\section{Table 1: Patient demographics}

\begin{tabular}{lc}
\hline Total patients & 32 \\
Median age in years (range) & $56(40-71)$ \\
Number female (\%) & $29(91)$ \\
Length of abobotulinumtoxin A use (years), $n(\%)$ & \\
$<2$ & $6(19)$ \\
$>2$ & $26(81)$ \\
Median abobotulinumtoxin A dose in glabella (SD) & $27.6( \pm 11.7)$ \\
Median incobotulinumtoxin A dose in glabella (SD) & $17.1( \pm 6.1)$ \\
Average conversion rate & $1.6: 1$ \\
Glabella re-treatment, $n$ (\%) (patient request) & \\
$\quad$ No & $29(91)$ \\
Yes & $3(9)$ \\
Pain versus abobotulinumtoxin A, $n$ (\%) & \\
(patient recall of abobotulinumtoxin A: questionnaire & \\
following incobotulinumtoxin A treatment) & \\
Equal & $20(63)$ \\
Greater & $7(22)$ \\
Less & $3(9)$ \\
Can't remember & $2(6)$ \\
\hline SD:Standard deviation
\end{tabular}

SD: Standard deviation perceived with the aboBTX-A dose, whereas 22\% had a less than expected response. Six percent of patients had a lesser response while receiving a relatively lower dose (ratio of 2.5-3:1) [Figure 1].

\section{DISCUSSION}

Injection of botulinum toxin type A was the most commonly performed cosmetic procedure in the Unites States in 2012, with over 4 million treatments reported for the year. ${ }^{[1]}$ Together with other injectable products, treatment with botulinum toxin type A accounted for approximately 2 billion dollars of Unites States spending in 2012. The rising number of minimally-invasive cosmetic procedures being performed, with a $10 \%$ increase in such procedures noted from 2011 to 2012, signals the long-term impact that injection of botulinum toxin type $A$ and other nonsurgical procedures will have on the future of aesthetic practices.

Reported overall patient satisfaction rates with botulinum toxin type A treatments are high ${ }^{[7,12]}$ and are largely related to the onset, duration, and efficacy of treatment. ${ }^{[7]}$ Given the tremendous impact of neuromodulator therapy and the importance of patient satisfaction as a key outcome, it is essential for practitioners to understand the optimal treatment dose, re-treatment interval, and expected outcomes associated with the various botulinum toxin $\mathrm{A}$ preparations available today.

Multiple clinical studies have demonstrated the safety, efficacy and tolerability of aboBTX-A since its Food and Drug Administration (FDA) approval in 2009 for the treatment of glabellar frown lines. ${ }^{[13-16]}$ A prospective, randomized control split-face trial comparing aboBTX-A with onaBTX-A found no significant differences between treatment effects on muscle activity or wrinkle appearance, onset and duration of treatment effect, or diffusion characteristics of the two neurotoxins. ${ }^{[8]}$ Although one clinical trial is comparing onaBTX-A with aboBTX-A indicated lower rates of patient satisfaction among patients treated with aboBTX-A, ${ }^{[17]}$ satisfaction rates with aboBTX-A treatments were high among our patient population. All our patients had previously received at least four consecutive treatments of aboBTX-A at 4-month intervals at a stable dose and had continued to achieve $85-90 \%$ elimination of dynamic glabellar frown lines at this dose with a reported high patient satisfaction rate.

Free of complexing proteins, incoBTX-A is unique among the botulinum toxin preparations currently available. The subtraction of these inactive or denatured protein

Table 2: Dose comparisons

\begin{tabular}{lcccc}
\hline & Incobotulinumtoxin A & Abobotulinumtoxin A & Mean difference (SD) (95\% Cl) & $\boldsymbol{P}^{\star}$ \\
\hline Dose in the glabella & $17.1(6.1)$ & $27.6(11.7)$ & $-10.5(8.6)$ & $(-13.6,-7.4)$ \\
Percentage of activity in glabella at & 20 & 27.5 & $-21.1(27.0)$ & $<0.001$ \\
$10-12$ weeks (per surgeon evaluation) & $69.2(27.3)$ & $90.3(1.8)$ & $(-30.8,-11.4)$ & $<.001$ \\
\hline
\end{tabular}

*Numbers in table are mean (SD) and median unless otherwise specified, difference calculated as incobotulinumtoxin A: abobotulinumtoxin $A$, $P$ value from paired t-test. SD: Standard deviation, Cl: Confidence interval 


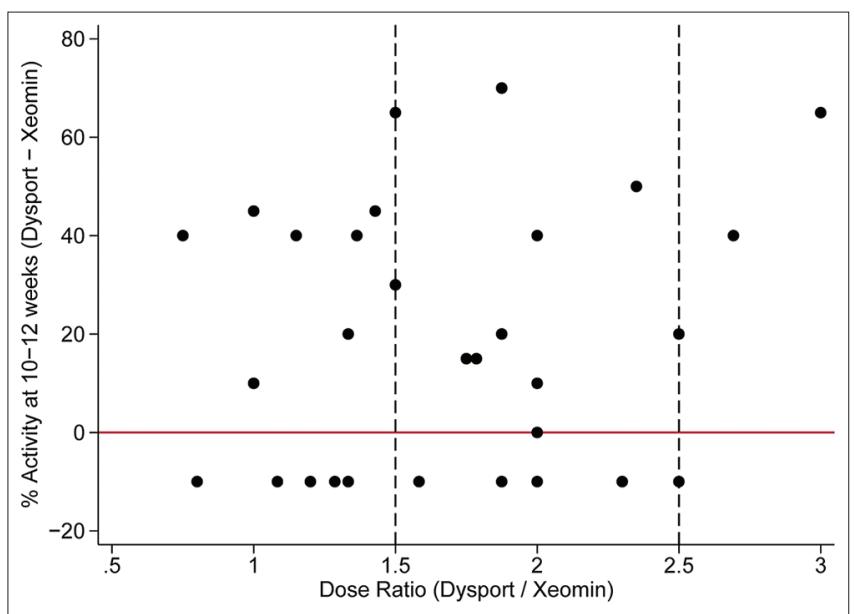

Figure 1: Neuromodulator dose ratio vs. muscle activity (percent) at 10-12 weeks

complexes theoretically results in a lower antigen load, decreasing the chance that the subject will develop neutralizing antibodies to treatment over time that could result in diminished clinical efficacy. ${ }^{[11,18]}$ IncoBTX-A received FDA approval for the treatment of glabellar frown lines in 2011, and a phase III clinical trial conducted that same year confirmed its efficacy for this indication, in accordance with FDA-mandated scoring criteria. ${ }^{[19]}$ One comparative trial of onaBTX-A and incoBTX-A reported overall high rates of patient satisfaction with both treatments and no statistically significant difference in satisfaction rates between the two neuromodulators. ${ }^{[20]} \mathrm{A}$ noninferiority trial comparing incoBTX-A with onaBTX-A found similar efficacy, safety, and patient satisfaction profiles between the two treatments at a 1:1 dosing ratio. ${ }^{[10]}$ No head-to-head trials comparing incoBTX-A with aboBTX-A have been published to date.

As such, our pilot study aimed to determine a dosing equivalency of incoBTX-A vs. aboBTX-A that would result in similar clinical effectiveness and patient satisfaction among 32 patients previously treated with aboBTX-A for at least four treatments over 1 year. At ratios believed to anticipate equivalent results based on prior dose comparison studies, ${ }^{[8-10]}$ we found that no precise ratio could be determined.

Among the patients who reported effect onset with incoBTX-A treatment, the median result was 8.5 days, longer than the median onset of effect with aboBTX-A treatment reported in the literature of 3 days. ${ }^{[21,22]}$

Limitations of our study include its small sample size, larger studies are warranted to better establish dose equivalency between aboBTX-A and incoBTX-A. IncoBTX-A injections were performed without cost to the patient, and this may have affected patient satisfaction rates. Secondary outcomes were determined through the use of a patient questionnaire. Recall bias may have affected our patients' ability to compare the pain of incoBTX-A injection compared with aboBTX-A injection which had occurred several months prior, and patient-reported onset and duration of effect may not be the most accurate means available of recording these results. However, given the elective nature of treatment to improve patient cosmesis, we believe that the patient report, while inherently biased, is still an acceptable method of outcome assessment.

In conclusion, the pilot study did not establish a dose equivalency between incobotulinumtoxin A (xeomin) vs. abobotulinumtoxin A (dysport) in the treatment of dynamic glabellar frown lines in 32 consecutive patients who previously reported treatment success with abobotulinumtoxin A for at least 1 year at 4-month intervals. By combining the analysis of both the patient-reported results and the objective evaluation of dynamic glabellar muscle activity at 10-12 weeks following one treatment session with incoBTX-A, we found that using incoBTX-A at $17.1( \pm 6.1)$ units was less predictable than using aboBTX-A at 27.6 ( \pm 11.7$)$ units. In comparison to aboBTX-A, the majority of our patients also reported lower satisfaction rates with incoBTX-A treatment; this difference was attributed to longer onset to treatment effect, increased pain on injection, and shortened duration of effect. Larger, prospective, randomized controlled studies are warranted to better establish dose equivalency between abobotulinumtoxin $\mathrm{A}$ and incobotulinumtoxin A.

\section{REFERENCES}

I. Cosmetic surgery national data bank: statistics 2012. Aesthet Surg / 2013;33: SI-2I.

2. Klein AW, Carruthers A, Fagien S, Lowe NJ. Comparisons among botulinum toxins:An evidence-based review. Plast Reconstr Surg 2008; I21:e4| 3-22.

3. Dressler D, Wohlfahrt K, Meyer-Rogge E, Wiest L, Bigalke H. Antibody-induced failure of botulinum toxin a therapy in cosmetic indications. Dermatol Surg 2010;36 Suppl 4:2182-7.

4. Ipsen Biopharmaceuticals, Medicis Aesthetics. Medication Guide Dysport (dis-port) (abobotulinumtoxinA) Injection. 2012. Availabe from: http://www.pi.medicis.us/medication_guide/dysport.pdf. [Last accessed on 2014 Jul I0].

5. Merz Pharmaceuticals, Merz Aesthetics. Medication Guide Xeomin (zeo-min) (incobotulinumtoxinA) for Injection, for Intramuscular Use. 201I. Available from: http://www.xeominaesthetic.com/ EM00674-XEOMIN-Medication-Guide.pdf. [Last accessed on 2014 Jul I0].

6. Matarasso A, Shafer D. Botulinum neurotoxin type A-ABO (Dysport): clinical indications and practice guide. Aesthet Surg J 2009;29:S72-9.

7. Flynn TC. Botulinum toxin: examining duration of effect in facial aesthetic applications. Am J Clin Dermatol 20 I0; I I:183-99.

8. Michaels BM, Csank GA, Ryb GE, Eko FN, Rubin A. Prospective randomized comparison of onabotulinumtoxinA (Botox) and abobotulinumtoxinA (Dysport) in the treatment of forehead, glabellar, and periorbital wrinkles. Aesthet Surg J 2012;32:96-102.

9. Freeman SR, Cohen JL. New neurotoxins on the horizon. Aesthet Surg J 2008;28:325-30.

10. Sattler G, Callander MJ, Grablowitz D, Walker T, Bee EK, Rzany B, Flynn TC, Carruthers A. Noninferiority of incobotulinumtoxinA, free from complexing proteins, compared with another botulinum toxin type $A$ in the treatment of glabellar frown lines. Dermatol Surg 2010;36 Suppl 4:2। 46-54.

II. Lorenc ZP, Kenkel JM, Fagien S, Hirmand H, Nestor MS, Sclafani AP, Sykes JM, Waldorf HA. IncobotulinumtoxinA (Xeomin): background, mechanism of action, and manufacturing. Aesthet Surg J 2013;33:SI8-22.

12. Fagien S, Carruthers JD. A comprehensive review of patient-reported satisfaction with botulinum toxin type a for aesthetic procedures. Plast Reconstr Surg 2008;122:1915-25.

13. Kane MA, Brandt F, Rohrich RJ, Narins RS, Monheit GD, Huber MB, Reloxin Investigational Group. Evaluation of variable-dose treatment with a new U.S. Botulinum Toxin Type A (Dysport) for correction of moderate to severe glabellar lines: results from a phase III, randomized, double-blind, placebo-controlled study. Plast Reconstr Surg 2009; 124:1619-29.

14. Baumann L, Brandt FS, Kane MA, Donofrio LM. An analysis of efficacy data 
from four phase III studies of botulinum neurotoxin type A-ABO for the treatment of glabellar lines. Aesthet Surg J 2009;29:S57-65.

15. Rubin M, Dover J, Maas C, Nestor M. An analysis of safety data from five phase III clinical trials on the use of botulinum neurotoxin type A-ABO for the treatment of glabellar lines. Aesthet Surg J 2009;29:S50-6.

16. Carruthers JD, Lowe NJ, Menter MA, Gibson J, Eadie N, Botox Glabellar Lines II Study Group. Double-blind, placebo-controlled study of the safety and efficacy of botulinum toxin type $A$ for patients with glabellar lines. Plast Reconstr Surg 2003; I I2:1089-98.

17. Lowe PL, Patnaik R, Lowe NJ.A comparison of two botulinum type a toxin preparations for the treatment of glabellar lines: double-blind, randomized, pilot study. Dermatol Surg 2005;31:165I-4.

18. PragerW. Differential characteristics of incobotulinumtoxin $A$ and its use in the management of glabellar frown lines. Clin Pharmacol 2013;5:39-52.

19. Carruthers A, Carruthers J. Commentary: long-term treatment of glabellar rhytides using onabotulinumtoxina. Dermatol Surg 201 I;37:929-30.

20. Prager W, Huber-Vorländer J, Taufig AZ, Imhof M, Kühne U, Weissberg R, Kuhr LP, Rippmann V, Philipp-Dormston WG, Proebstle TM, Roth C,
Kerscher M, Ulmann C, Pavicic T. Botulinum toxin type A treatment to the upper face: retrospective analysis of daily practice. Clin Cosmet Investig Dermatol 20I2;5:53-8.

21. Moy R, Maas C, Monheit G, Huber MB, Reloxin Investigational Group. Long-term safety and efficacy of a new botulinum toxin type $A$ in treating glabellar lines. Arch Facial Plast Surg 2009; I I:77-83.

22. Brandt F, Swanson N, Baumann L, Huber B. Randomized, placebo-controlled study of a new botulinum toxin type a for treatment of glabellar lines: efficacy and safety. Dermatol Surg 2009;35:1893-90I.

How to cite this article: Bank J, Phillips NA, Casas LA. Patientcentric dose equivalency pilot study of incobotulinumtoxin a (xeomin) $v s$. abobotulinumtoxin a (dysport) in the treatment of glabellar frown lines. Plast Aesthet Res 2015;2:12-6.

Source of Support: Nil, Conflict of Interest: None declared.

Received: 24-07-2014; Accepted: 28-09-2014 